\title{
Representation of model data and evaluation of diagnostic equations in pressure coordinates
}

\author{
By PIERLUIGI CALANCA ${ }^{\mathbf{1}}$, and CARL FORTELIUS ${ }^{2 *}{ }^{1}$ Swiss Federal Laboratories for Materials \\ Testing and Research, Überlandstr. 129, CH-8600 Dübendorf, Switzerland, ${ }^{2}$ Department of Meteorology, \\ Box 4 (Yliopistonkatu 3), FIN-00014 University of Helsinki, Finland
}

(Manuscript received 13 October 1995; in final form 11 March 1996)

\begin{abstract}
This article presents a method for transforming atmospheric model data to isobaric coordinates in such a way that mass-weighted integrals are conserved and that the variable surface pressure is naturally treated. The transformation is based on integrating model variables over finite pressure intervals. Representative values at isobaric levels are defined in terms of these integrals. Evaluation of atmospheric budget equations in pressure coordinates using transformed data is discussed on the example of the thermodynamic energy equation. Layer-integrated budgets calculated in model coordinates may be satisfactorily reproduced if the layout of the target system is carefully designed. In the specific case treated, the most delicate component is the energy conversion term, which can be accurately evaluated in the isobaric frame only if the vertical resolution is comparable to that of the model system. Nevertheless, good quantitative estimates of vertical integrals and qualitatively reasonable three-dimensional distributions of individual terms are obtained even after a significant reduction of the vertical resolution. The transformation scheme is conceptually simple and easy to implement. The method is applicable to transformations between arbitrary coordinate systems.
\end{abstract}

\section{Introduction}

An increasing number of empirical investigations on the atmosphere is being based on archives of meteorological data assimilation products (Schubert et al., 1993; Gibson et al., 1994). Most studies rely on the availability of data bases of a manageable size, especially for investigations covering extended periods of time. As most forecast models are now running with more than 20 levels, a significant reduction of the data volume is automatically realized by moving from the model frame to a standard pressure system consisting of some 10 to 15 levels, a procedure included in operational postprocessors.

\footnotetext{
* Corresponding author. email: carl.fortelius@helsinki.fi
}

The adoption of the isobaric system may be largely due to historical reasons, but there are distinct advantages still valid to date (Trenberth, 1995). In particular, contrary to model coordinate systems, the isobaric frame is formally independent of the surface pressure, and therefore well suited for creating archives of so-called circulation statistics.

Operational transfer schemes are based on local interpolation, typically linear or logarithmic in pressure. They yield accurate level values for the specified pressure surfaces, but these values are not representative for finite sized layers (Trenberth, 1991). Thus, integral quantities, such as the precipitable water, are generally not preserved by the transformation. A related problem of many operational schemes is that they create an artificial atmosphere by extrapolating fields below ground. Later, care must be taken that such 


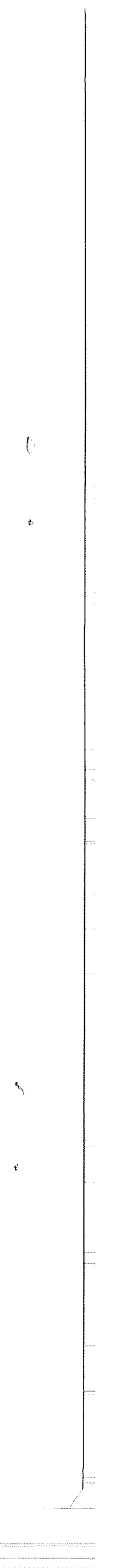


spurious data are not propagating into diagnostic results. This can be attained by introducing an auxiliary variable, as elaborated by Boer (1982). Of course this problem is related to the fundamental drawback of the isobaric system, namely that earth's surface does not coincide with a coordinate surface. Both of these problems can be elegantly solved by considering integrals over finite pressure layers, as defined in terms of the untransformed fields, instead of local level values.

Yet another disadvantage of operational postprocessing routines is that diagnostic relationships which hold in the model frame are no longer satisfied when variables are interpolated independently of each other. This is most evident in the budget of mass (Savijärvi, 1982), but is potentiaily a large source of error in all budgets calculated using transformed data because all such evaluations implicitly assume that mass is conserved. Fortunately, the fact that there exist no real sources and sinks of dry air makes it possible to restore the mass balance with an appropriate modification of the fields of motion (Trenberth et al., 1995). Another possibility to ensure a state of mass balance in the output frame is to compute the vertical velocity directly from the transformed horizontal mass flux. This vertical velocity field is consistent with the original one only if the transformation conserves the vertically-integrated mass flux and if the same physical boundary conditions are applied in both reference frames.

One may question the utility of being able to compute budgets in an alternative frame, since, in principle, all terms could always be evaluated in the original system of coordinates and transformed subsequently. As far as we are aware, data analysis centres have not yet been ready to include individual terms of budget equations in their archives. Moreover, even if this could be realized for some quantities, an algorithm for computing budgets in pressure coordinates would still be useful for investigations based on filtered data.

In Section 2 of the present article, we describe an algorithm for transforming model data to the isobaric system. The scheme is based on computing integrals over finite layers. In Section 3, we discuss evaluating atmospheric budget equations, as exemplified by the thermodynamic energy equation, with the aid of layer integrals of the state variables. In approximating layer integrals of nonlinear terms by nonlinear combinations of the transformed variables, one neglects the correlations arising from the variability of the original fields within the pressure layers. The magnitude of the resulting error depends on the layout of the target system, and is the subject of Section 4 . We explore this dependence by comparing estimates computed in the pressure system to reference values, obtained by diagnosing the same physical quantities by their appropriate expressions in the model frame, and then transposing the results into the pressure frame.

The work reported on here is the first part of an undertaking to assess the utility of products of the HIRLAM data assimilation system (Andersson and Gustafsson, 1994) in investigations of the atmospheric cycles of energy and water over the Baltic Sea area. Although we present a procedure adapted to a particular weather prediction system, our results should be applicable quite generally.

\section{The transformation algorithm}

The HIRLAM model employs a hybrid vertical coordinate $(\eta)$, that follows the terrain at low levels and approaches the isobaric system above. Except for the surface pressure, prognostic variables (horizontal wind, temperature, specific humidity and liquid water content) are carried at $N$ full levels, representing $N$ layers bounded by $N+1$ half levels. The vertical velocity $\dot{\eta}$ is diagnosed at the layer boundaries. The pressure at the half levels is given by:

$p\left(\eta_{n+1 / 2}\right)=A_{n+1 / 2}+B_{n+1 / 2} p_{\mathrm{s}}, n=0,1, \ldots, N$,

where $p_{\mathrm{s}}$ is the surface pressure and the coefficients $A_{n+1 / 2}$ and $B_{n+1 / 2}$ are specified so that $p\left(\eta_{1 / 2}\right)=0$ and $p\left(\eta_{N+1 / 2}\right)=p_{s}$.

The layout of the constant pressure grid and the distribution of variables are analogous to those of the model grid. There are $K$ layers represented by $K$ full levels and delimited by $K+1$ half levels; $p_{1 / 2}$ is at $0 \mathrm{hPa}$, whereas the lowest half level pressure is chosen sufficiently large so that the relation $p_{\mathrm{K}+1 / 2}>p_{\mathrm{s}}$ is always satisfied.

The primary requirement of our transformation procedure is the conservation of vertical integrals. The problem thus reduces to expressing integrals over pressure-layers in terms of the original level

Tellus 48A (1996), 5 
values. The discretization of the continuity and hydrostatic equations in the model formulation (Simmons and Burridge, 1981) implies that prognostic variables are representative of their respective layer. We can therefore define a massweighted integral $\tilde{\xi}_{\mathrm{n}}$ of a model variable $\xi$ over a model layer $n$ as:

$\tilde{\xi}_{n}=\xi_{n}\left[p\left(\eta_{n+1 / 2}\right)-p\left(\eta_{n-1 / 2}\right)\right]$.

The mass-weighted integral $\hat{\xi}_{k}$ of $\xi$ over an arbitrary layer $k$ of the isobaric frame is then obtained as a linear combination of the layer integrals $\tilde{\xi}_{n}$ :

$\hat{\xi}_{k}=\sum_{n=1}^{N} w_{k n} \tilde{\xi}_{n}$.

Assuming the model variables to be constant within the layers, the coefficients $w_{k n}$ are simply given by the ratio of the part of the model layer $n$ overlapping the isobaric layer $k$ to the total depth of the model layer, that is:

$$
w_{k n}= \begin{cases}0 & \text { if } p_{k-1 / 2} \geqslant p\left(\eta_{n+1 / 2}\right), \\ \frac{p\left(\eta_{n+1 / 2}\right)-p_{k-1 / 2}}{p\left(\eta_{n+1 / 2}\right)-p\left(\eta_{n-1 / 2}\right)} & \text { if } p\left(\eta_{n-1 / 2}\right)<p_{k-1 / 2} \\ & <p\left(\eta_{n+1 / 2}\right) \leqslant p_{k+1 / 2}, \\ \frac{p_{k}+1 / 2}{p\left(\eta_{n+1 / 2}\right)-p\left(\eta_{n-1 / 2}\right)} & \text { if } p_{k-1 / 2}>p\left(\eta_{n-1 / 2}\right) \\ & \text { and } \\ & p_{k+1 / 2}<p\left(\eta_{n+1 / 2}\right), \\ & \text { if } p_{k-1 / 2} \leqslant p\left(\eta_{n-1 / 2}\right) \\ & \text { and } \\ & p_{k+1 / 2} \geqslant p\left(\eta_{n+1 / 2}\right), \\ \frac{p_{k+1 / 2}-p\left(\eta_{n-1 / 2}\right)}{p\left(\eta_{n+1 / 2}\right)-p\left(\eta_{n-1 / 2}\right)} & \text { if } p_{k-1 / 2} \leqslant p\left(\eta_{n-1 / 2}\right) \\ & <p_{k+1 / 2}<p\left(\eta_{n+1 / 2}\right), \\ 0 & \text { if } p_{k+1 / 2} \leqslant p\left(\eta_{n-1 / 2}\right) .\end{cases}
$$

Eqs. (3) and (4) completely determine the transformation of model data to isobaric coordinates and in principle nothing more is needed. However, we find it in order to discuss some important properties of the transformation scheme. We begin by remarking that the coefficients satisfy the following relations:

$0 \leqslant \sum_{n=1}^{N} w_{k n} \leqslant N, \forall k$ and

$\sum_{k=1}^{K} w_{k n}=1, \forall n$

Consequently mass-weighted integrals over the whole atmospheric column are conserved exactly regardless of the value of $K$, since:

$$
\begin{aligned}
\sum_{k=1}^{K} \hat{\xi}_{k} & =\sum_{k=1}^{K} \sum_{n=1}^{N} w_{k n} \tilde{\xi}_{n}=\sum_{n=1}^{N} \tilde{\xi}_{n} \sum_{k=1}^{K} w_{k n} \\
& =\sum_{n=1}^{N} \tilde{\xi}_{n} .
\end{aligned}
$$

The specific assumption concerning the variation of the variables between model levels will of course to some extent influence the contribution of a given model level value to a given layer integral and therefore the vertical distribution of the fields in the pressure frame.

Eqs. (3) and (4) show that the layer integrals remain well-defined when the layer is intercepted by the surface and even when the layer is entirely below the surface, in which latter case the integral automatically vanishes. This important characteristic of the transformation recalls the action of the multiplier $\beta$ introduced by Boer (1982) for level data, and modified by Trenberth (1991) to cope with finite sized layers. We can recover Trenberth's $\beta$ by applying (3) to the unit function:

$\hat{\mathbf{1}}_{k}=\sum_{n=1}^{N} w_{k n} \tilde{\mathbf{1}}_{n}$,

where

$\tilde{\mathbf{1}}_{n}=p\left(\eta_{n+1 / 2}\right)-p\left(\eta_{n-1 / 2}\right)$.

Since $\hat{\mathbf{1}}_{\mathrm{k}}$ is the physical depth of the layer $k$, it is easily demonstrated that:

$$
\begin{aligned}
\frac{\hat{\mathbf{1}}_{k}}{p_{k+1 / 2}-p_{k-1 / 2}} & =\beta_{k} \\
& = \begin{cases}1 & \text { if } p_{k+1 / 2} \leqslant p_{\mathrm{s}}, \\
\frac{p_{\mathrm{s}}-p_{k-1 / 2}}{p_{k+1 / 2}-p_{k-1 / 2}} & \text { if } p_{k-1 / 2}<p_{\mathrm{s}} \\
& <p_{k+1 / 2}, \\
0 & \text { if } p_{k-1 / 2} \geqslant p_{\mathrm{s}} .\end{cases}
\end{aligned}
$$

Using the physical layer depth it is easy to compute representative layer averages of a vari-

Tellus 48A (1996), 5 
able $\xi$ :

$\xi_{k}= \begin{cases}\frac{\hat{\xi}_{k}}{\hat{\mathbf{1}}_{k}} & \text { if } \hat{\mathbf{1}}_{k}>0 \\ \text { arbitrary } & \text { otherwise. }\end{cases}$

For moderately thick layers, the layer averages may also serve as excellent approximations for local values. The particular value assigned to the representative averages of layers entirely below the ground can be chosen according to one's purposes. In the present context, we leave it arbitrary, but finite, because it does not influence diagnostic results when budget relations are evaluated according to the scheme presented in Section 3.

It will be seen in the next section that it is convenient to include a definition of surface values $\xi_{\mathrm{s}}$ in the transformation scheme. The definition of these values must depend on the specific circumstances. For the computations presented in Section 4 , we simply take the values at the lowest model level.

We close this section by two remarks. The first concerns a computational aspect of the transformation scheme. Because the model's hybrid levels are a function of the surface pressure, the same holds true also for the coefficients of the transformation $w_{k n}$. In principle they should thus be calculated for each horizontal grid point and at each time. In practice we have found it expedient and sufficiently accurate to recover them by linear interpolation from a look-up table containing precomputed coefficients for an adequate range of surface pressure values. The retrieval is efficient because hunting in the table can be started either from the position of a neighbour grid point or from the position at a previous time step. This makes the computational demand of our transformation scheme comparable to that of algorithms based on linear interpolation.

As a second and final remark, we wish to point out that the procedure outlined here is not restricted to transforming data from the hybrid coordinate system to the isobaric system, but is applicable quite generally. The only requirement is that the integrals over suitable intervals of the target coordinate may be accurately expressed in terms of the original fields.

\section{Diagnostics in pressure coordinates: evaluation}

Evidently, the pressure coordinate representation of any budget equation suffering the least from a change of the frame of reference is the one obtained by transforming the diagnostics computed in the model framework. However, in the event that the budget one wishes to study is not included in the applied postprocessing, it becomes necessary to retrieve it directly in the pressure frame from fields that have been previously transformed. Naturally, the accuracy of any such approximation will depend on the layout and vertical resolution of the target frame. We will return to this issue in Section 4. Here we present a finite difference scheme suited to our representation of isobaric data as integrals over finite sized pressure layers and discuss some general features. We derive the scheme specifically for the thermodynamic energy equation, written in the form:

$\frac{\partial}{\partial t_{\mathrm{p}}} h+v \cdot \nabla_{\mathrm{p}} h+\omega \frac{\partial h}{\partial p}-\alpha \omega=J$

where $h=c_{\mathrm{p}} T$ denotes the dry enthalpy per unit mass, $c_{\mathrm{p}}$ is the specific heat at constant pressure, $T$ is the temperature, $\alpha$ is the specific volume, $J$ is the diabatic heating rate and, finally, $\partial / \partial t_{\mathrm{p}}$ and $\nabla_{\mathrm{p}}$ are the isobaric local tendency and gradient operators.

We choose to treat this particular equation partly because of its general interest, but also because it is highly sensitive to small errors in the data (Holopainen and Fortelius, 1986). Yet another reason to examine this particular form of the thermodynamic energy equation is that a specific formulation of the energy conversion term $\alpha \omega$ is provided in the model, and we wish to find out how well this particular term can be reproduced. The discussion concerning the local tendency and horizontal and vertical advection can, of course, be immediately extended to any budget equation.

The integral of the local tendency can be expressed in terms of the integrated enthalpy without particular assumptions. Invoking Leibniz' rule we have:

$\left(\frac{\widehat{\partial h}}{\partial t_{\mathrm{p}}}\right)_{k}=\frac{\partial}{\partial t} \hat{h}_{k}+h_{\mathrm{s}} \tau_{k}$

Tellus 48A (1996), 5 
where the boundary term $\tau_{k}$ is given by:

$\tau_{k}= \begin{cases}0 & \text { if } p_{k+1 / 2}<p_{s}, \\ -\frac{\partial p_{s}}{\partial t} & \text { if } p_{k-1 / 2}<p_{s} \leqslant p_{k+1 / 2}, \\ 0 & \text { if } p_{k-1 / 2} \geqslant p_{s},\end{cases}$

and where $h_{\mathrm{s}}$ denotes the value of the specific enthalpy at the surface. The surface pressure tendency is computed as:

$\frac{\partial p_{\mathrm{s}}}{\partial t}=-\nabla \cdot \sum_{k=1}^{K} \hat{\boldsymbol{v}}_{k}$,

a formulation which, according to (6), yields exactly the same numerical value as one would obtain in the model framework.

The horizontal advection is more problematic in two respects. First, we need a simple and wellbehaved representation valid even for layers affected by the earth's surface. This is best achieved by restating the advection with the aid of the flux divergence according to:

$\left(\widehat{\boldsymbol{v} \cdot \nabla_{\mathrm{p}} h}\right)_{k}=\left(\widehat{\nabla_{\mathrm{p}} \cdot h \boldsymbol{v}}\right)_{k}-\left(\widehat{h \nabla_{\mathrm{p}} \cdot \boldsymbol{v}}\right)_{k}$

or, applying again Leibniz' rule:

$\left.\widehat{\left(\boldsymbol{v} \cdot \nabla_{\mathrm{p}} h\right.}\right)_{k}=\nabla \cdot(\widehat{h v})_{k}+h_{\mathrm{s}} \lambda_{k}-\left(\widehat{h \nabla_{\mathrm{p}} \cdot \boldsymbol{v}}\right)_{k}$.

In expressing the advection as in (16) we take advantage of the fact that the layer-integrated enthalpy flux is defined in every circumstance, and the same holds true for its divergence. However, we are still left with the second, and more fundamental problem of the nonlinearity of the advective term. In the case that only the variables of state are transformed, the first and third terms on the r.h.s of (16) are not available directly in the isobaric frame. Here, we resort to the following approximation, which neglects the vertical variation of wind and temperature within the layers:

$\left.\widehat{\left(v \cdot \nabla_{\mathrm{p}} h\right.}\right)_{k}=\nabla \cdot h_{k} \hat{v}_{k}+h_{\mathrm{s}} \lambda_{k}+h_{k} \Delta \omega_{k}$,

where $h_{k}$ is the layer-averaged enthalpy as defined by (10) and $-\Delta \omega_{k}$ is the layer integrated horizontal mass flux divergence, calculated in exact analogy with the enthalpy flux divergence as:

$\Delta \omega_{k}=-\nabla \cdot \hat{v}_{k}-\lambda_{k}$.
Analytically, the boundary term $\lambda_{\mathrm{k}}$ is given by:

$\lambda_{k}= \begin{cases}0 & \text { if } p_{k+1 / 2}<p_{\mathrm{s}}, \\ -v_{\mathrm{s}} \cdot \nabla p_{\mathrm{s}} & \text { if } p_{k-1 / 2}<p_{\mathrm{s}} \leqslant p_{k+1 / 2}, \\ 0 & \text { if } p_{k-1 / 2} \geqslant p_{\mathrm{s}},\end{cases}$

where $v_{\mathrm{s}}$ denotes the horizontal wind at the surface. An algorithm suitable for the discrete case is presented in the Appendix.

To match the formulation of the horizontal advection, the vertical advection is restated as:

$$
\begin{aligned}
{\left[\overline{\left.\omega \frac{\partial h}{\partial p}\right]_{k}=}\right.} & \left(h_{k+1 / 2} \omega_{k+1 / 2}-h_{k-1 / 2} \omega_{k-1 / 2}\right) \\
& -h_{k} \Delta \omega_{k} .
\end{aligned}
$$

We derive the half level values of the specific enthalpy by requiring that, for layers not affected by the surface, our procedure should be equivalent to a scheme employed in many weather prediction models, including HIRLAM, because of its conservation properties (Haltiner and Williams, 1980). This leads to:

$h_{k+1 / 2}= \begin{cases}\frac{1}{2}\left(h_{k}+h_{k+1}\right) & \text { if } p_{k+1 / 2}<p_{\mathrm{s}}, \\ \frac{1}{2}\left(h_{k}+h_{\mathrm{s}}\right) & \text { if } p_{k-1 / 2}<p_{\mathrm{s}} \leqslant p_{k+1 / 2}, \\ h_{\mathrm{s}} & \text { if } p_{k-1 / 2} \geqslant p_{\mathrm{s}},\end{cases}$

Keeping the value of $h$ constant below the surface is a simple way to guarantee that the vertical flux divergence vanishes there.

The values of $\omega$ at the layer boundaries are already defined by the transformed horizontal mass flux through the continuity eq. (18):

$\omega_{k+1 / 2}=\omega_{1 / 2}+\sum_{j=1}^{k} \Delta \omega_{j}$.

With the boundary condition $\omega_{1 / 2}=0, \omega_{k+1 / 2}$ satisfies the condition:

$\omega_{k+1 / 2}=\omega_{\mathrm{s}}=\frac{\partial p_{\mathrm{s}}}{\partial t}+v_{\mathrm{s}} \cdot \nabla p_{\mathrm{s}}$,

$$
\text { if } p_{k-1 / 2}<p_{\mathrm{s}} \leqslant p_{k+1 / 2},
$$

where the surface pressure tendency is again given by (14). Further, inspection of (18) and (19) shows that below the earth's surface $\omega$ formally remains constant at the surface value. This is coherent with a vanishing mass flux there.

Approximating (16) by (17) is not dictated

Tellus 48A (1996), 5 
merely by practical considerations. The fundamental reason is the need to make the numerics employed for the advective terms matching those used for the continuity equation (18) and to achieve a complete equivalence between the threedimensional advection and three-dimensional flux divergence. When this has been attained, the sums of these terms over all the layers satisfy the relation:

$\left.\sum_{k=1}^{K}\left\{\widehat{\left(v \cdot \nabla_{p} h\right.}\right)_{k}+\left(\widehat{\omega \frac{\partial h}{\partial p}}\right)_{k}\right\}=\nabla \cdot \sum_{k=1}^{K} h_{k} \hat{v}_{k}+h_{\mathrm{s}} \frac{\partial p_{\mathrm{s}}}{\partial t}$,

so that:

$$
\begin{gathered}
\sum_{k=1}^{K}\left\{\left(\frac{\widehat{\partial h}}{\partial t_{\mathrm{p}}}\right)_{k}+\widehat{\left(\widehat{v} \cdot \nabla_{\mathrm{p}} h\right.}\right)_{k}+\left(\widehat{\left.\left.\omega \frac{\partial h}{\partial p}\right)_{k}\right\}}\right. \\
=\frac{\partial}{\partial t} \sum_{k=1}^{K} \hat{h}_{k}+\nabla \cdot \sum_{k=1}^{K} h_{k} \hat{v}_{k} .
\end{gathered}
$$

Hence vertically-integrated budgets over the whole atmosphere are not influenced by the formulation chosen for the vertical enthalpy flux, but only by the approximate formulation of the layerintegrated horizontal flux.

We come finally to the last term in the enthalpy budget. The computation of the energy conversion term $\alpha \omega$ does not require further assumptions or approximations. Keeping the temperature and wind constant and integrating over a layer gives, making use of the equation of state $p \alpha=R T$ :

$(\widehat{\alpha \omega})_{k}=$

$$
\left\{\begin{array}{c}
\frac{R}{c_{\mathrm{p}}} h_{k}\left[\begin{array}{c}
\Delta \omega_{k}+\left(\omega_{k-1 / 2}-p_{k-1 / 2} \frac{\Delta \omega_{k}}{\hat{\mathbf{1}}_{k}}\right) \\
\times \ln \left(\frac{p_{k+1 / 2}}{p_{k-1 / 2}}\right)
\end{array}\right] \begin{array}{l}
\text { if } p_{k+1 / 2} \\
<p_{\mathrm{s}}
\end{array} \\
\frac{R}{c_{\mathrm{p}}} h_{k}\left[\begin{array}{cc}
\left.\Delta \omega_{k}+\left(\omega_{k-1 / 2}-p_{k-1 / 2} \frac{\Delta \omega_{k}}{\hat{\mathbf{1}}_{k}}\right)\right] \\
\times \ln \left(\frac{p_{\mathrm{s}}}{p_{k-1 / 2}}\right)
\end{array}\right] \begin{array}{l}
\text { if } p_{k-1 / 2} \\
<p_{\mathrm{s}} \\
\leqslant p_{k+1 / 2}, \\
0
\end{array} \\
\\
\end{array}\right.
$$

where $R$ is the gas constant for air. The expression (26) is the pressure coordinate form of the expres- sion derived by Simmons and Burridge (1981). Note that the terms involving the logarithmic layer depth vanish in the top layer due to the boundary conditions at the top of the atmosphere.

\section{Diagnostics in pressure coordinates: validation}

We apply the transformation scheme to data from a re-assimilation experiment carried out by SMHI, the Swedish Meteorological and Hydrological Institute, based on the HIRLAM limited area data assimilation system (Ljungemyr et al., 1996). A concise description of the HIRLAM model is given by Andersson and Gustafsson (1994). It is a grid-point limited area model employing Arakawa-C horizontal staggering. The computational domain is centred over Norway at about $63^{\circ} \mathrm{N}$ and $6^{\circ} \mathrm{E}$. The grid consists of 162 points in the zonal and 142 points in the meridional direction, respectively, with a mesh size of approximately $22 \mathrm{~km}$. The vertical discretization is realized with 24 hybrid layers.

In order to isolate the consequences of the vertical transformation, we compute isobaric diagnostics of the thermodynamic energy equation keeping the same horizontal grid structure and attendant numerics. We conduct the analysis for just one short-range forecast, valid at 12 UTC, 18 December 1986. Doing so, we cannot study explicitly the enthalpy tendency, but we have shown in Section 3 that an exact representation is possible for this particular term. Moreover, since the model solution is relaxed towards external boundary values near the edges of the domain, we omit the outermost 20 gridpoints along all four boundaries. We design three sets of constant pressure layers suited to investigate the implications of a reduction of the vertical resolution. The first set has 21 layers, defined by the 22 half level values $0,14,44,88,142,204,271,341,412,482$, $550,615,675,730,779,824,864,904,944,984$, 1024 and $1064 \mathrm{hPa}$, chosen to coincide as close as possible with the model layers, except in the lower troposphere, where the model vertical coordinate becomes terrain-following. This set, labelled L21, ought to ensure a minimum loss of accuracy. The second and third sets have 16 and 9 layers. They are denoted by $\mathrm{L} 16$ and L9, and are defined by the half levels $0,14,44,88,140,210,285,365,455$,

Tellus 48A (1996), 5 
$550,650,750,830,900,965,1025,1075 \mathrm{hPa}$ and $0,50,150,275,400,525,650,800,950,1100 \mathrm{hPa}$, respectively. In the lower troposphere, the layout of these two latter sets is not too far from the one of the standard isobaric system employed in most operational postprocessors.

For each of the three layer configurations, we provide reference fields by evaluating the enthalpy advection and the energy conversion term within the model framework making use of the appropriate expressions for $\nabla_{\mathrm{p}}$ and $\partial / \partial t_{\mathrm{p}}$, and transforming the result. We quantify the departure from this reference by the area-mean error and by the standard deviation of the error (Sass and Christensen, 1995), whereas we describe the fields themselves by their area average and standard deviation.

Fig. 1 shows vertical profiles of the descriptive statistics for the horizontal and vertical enthalpy advection, the energy conversion term and the difference between the three-dimensional advection and the energy conversion. This latter, combined with the enthalpy tendency, would yield an estimate of the apparent diabatic heating (see eq. 11). In the case studied, which was characterized by vigorous synoptic activity with, in particular, a deep cyclone between Scotland and Norway and an associated strong easterly flow over the Scandinavian mountains, the energy conversion is mainly balanced by vertical enthalpy advection in the middle troposphere and by horizontal enthalpy advection in the stratosphere. Note that the very large mean and standard deviation of the horizontal enthalpy advection and energy conversion in the L21 and L16 sets above $100 \mathrm{hPa}$ are not an artefact of the transformation, since this latter is trivial when model and pressure layers coincide. The proof is provided by Fig. 2, which
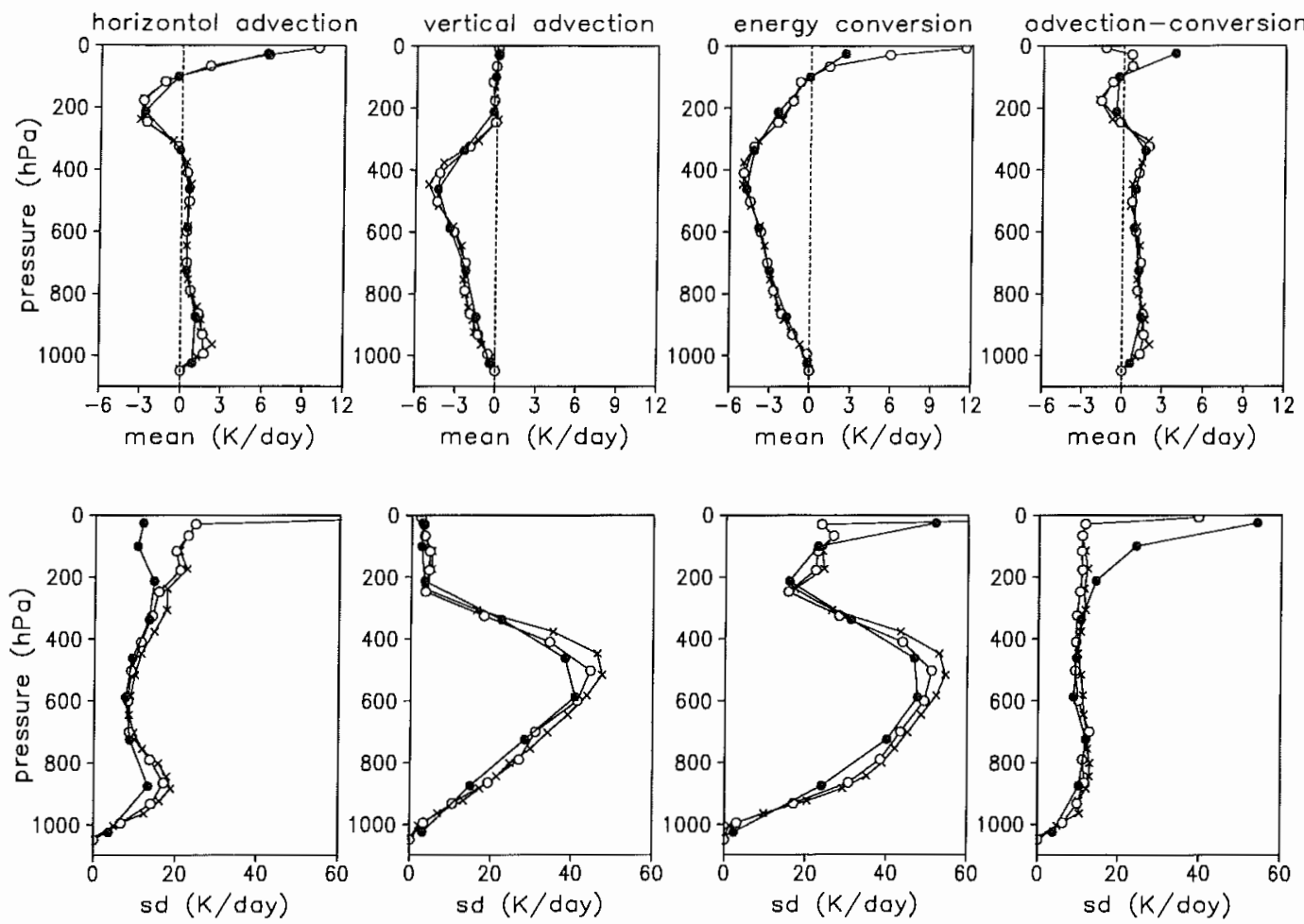

Fig. 1. Vertical distribution of the area mean and standard deviation (sd) of the enthalpy advection, the energy conversion term and their difference, as evaluated using transformed winds and temperature. Both statistics are divided by the nominal layer thickness and are shown in units of $\mathrm{K}_{\text {day }}{ }^{-1}$. The crosses, open and full circles pertain to the configurations L21, L16 and L9, respectively.

Tellus 48A (1996), 5 

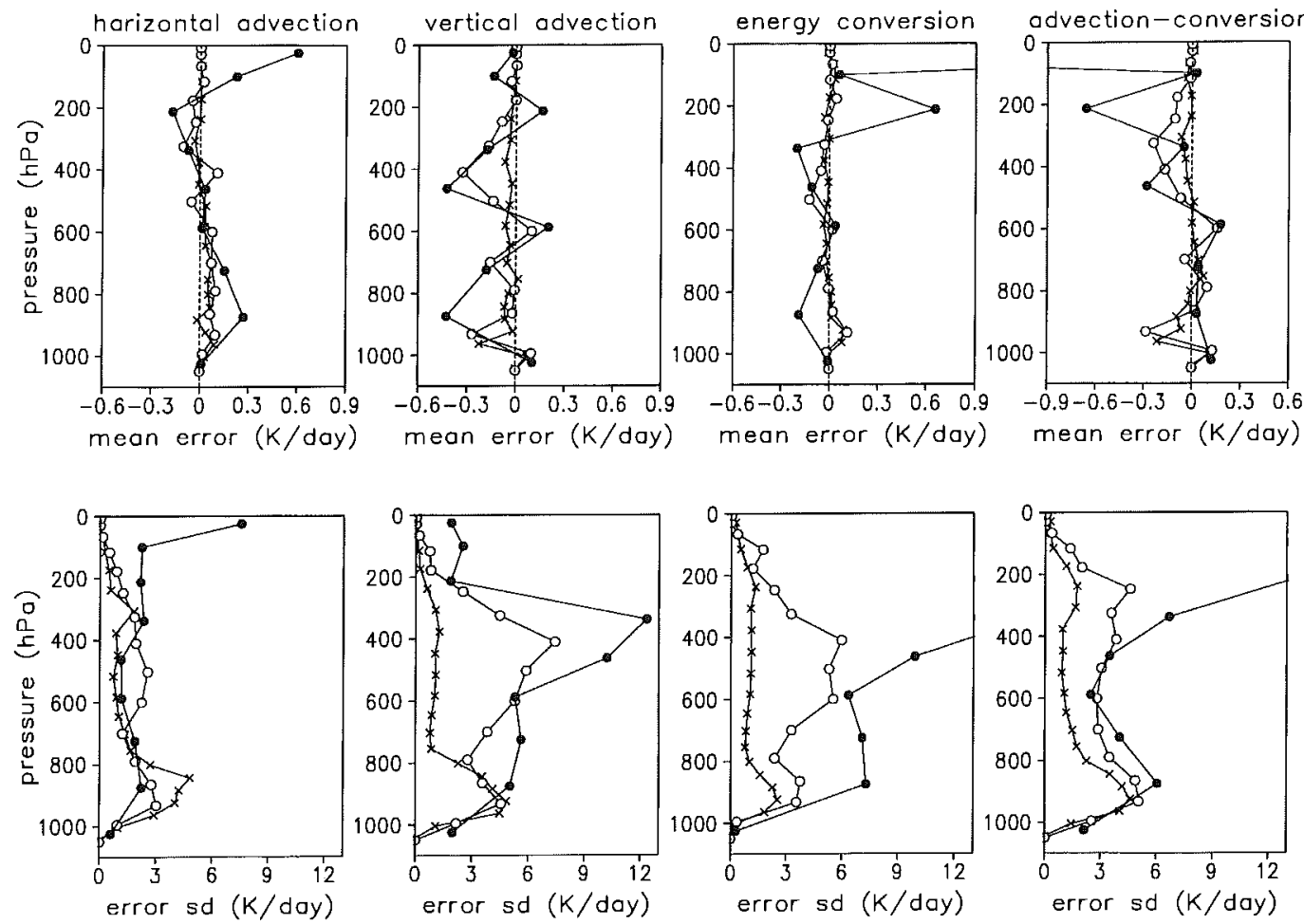

Fig. 2. Vertical distribution of the area mean and standard deviation (sd) of the error in the isobaric estimate of the enthalpy advection, the energy conversion term and their difference, defined as the departure of the approximate value from the reference value obtained in the model framework. Both statistics are divided by the nominal layer thickness and are shown in units of $\mathrm{K} \mathrm{day}^{-1}$. The crosses, open and full circles pertain to the configurations L21, L16 and L9, respectively.

indicates that for the L21 and L16 configurations the standard deviation of the error is in fact negligibly small above $100 \mathrm{hPa}$.

As seen further in Fig. 2, with 21 layers a significant standard deviation of the error occurs, for all fields, only in the lower troposphere, where the model grid and the pressure grid are indeed different entities.

Of the three terms considered separately, the horizontal advection displays the least sensitivity to the layout of the vertical grid. Almost paradoxically, decreasing the vertical resolution in the lower troposphere improves the accuracy of the results. The reason is that the small-scale noise near steep orography is partially filtered out when the layer thickness is increased.

In the middle and upper troposphere, the accuracy of the vertical enthalpy advection drops substantially as the vertical resolution decreases.
This must be directly related to the specification of the temperature at the layer boundaries for evaluating (21). Actually, the vertical advection is not only sensitive to the layer thickness itself but even more so to abrupt thickness changes between any two adjacent layers.

The accuracy of the isobaric representation of the energy conversion term does also show a strong dependence on the vertical grid structure, although it is formally calculated under the same assumptions as the horizontal enthalpy advection, namely those of constant temperature and wind within the layers. The deep layers employed in the L9 configuration are entirely inadequate for the computation of this term. The reason must be a relatively high level of correlation in the vertical dimension between sub-grid scale variability of the temperature and the vertical velocity.

The comparison of the standard deviation of

Tellus 48A (1996), 5 
the error to that of the fields themselves shows that for the L16 set the ratio of these two rarely exceeds a value of 0.3 for any of the single terms. It seems thus possible to obtain a good qualitative depiction of the three-dimensional distribution of the single terms appearing in the thermodynamic energy equation using transformed data despite a marked reduction in the number of layers.

The situation is more critical for the difference between three-dimensional enthalpy advection and energy conversion, due to the close balance of the two fields. Remember that the accuracy of this difference can be directly interpreted as the accuracy of the isobaric estimate of the diabatic heating. For the L16 and L9 configurations the standard deviation of the error is almost as large as that of the field itself, a fact which renders the quantitative evaluation of the diabatic heating hopeless at these resolutions.

The vertically-integrated enthalpy budget is somewhat less sensitive. As suggested by the statistics of Table 1, for the case studied a mean relative error of about $5 \%$ should be expected for the total diabatic heating with the L16 configuration, whereas locally the relative error can attain some $30 \%$. The numbers in Table 1 indicate further that, at the $\mathrm{L} 9$ resolution, the mean relative error of the total diabatic heating can increase to $30 \%$, with local maxima up to $100 \%$.

As could be expected based on the discussion concerning (25), Table 1 shows that the verticallyintegrated enthalpy flux divergence is excellently reproduced even with merely nine layers. Very likely, this finding can be extended to the flux divergence of other fields. Consequently, even after substantially reducing the vertical resolution, it should be possible to obtain excellent estimates of the moisture budget, which does not contain any term analogous to the energy conversion term in the enthalpy equation.

Finally we would like to point out that even the reference values may depart significantly from the physical truth. Such a departure can be due to a combination of data and model errors. For instance, the sensitivity to the vertical resolution noted in the energy conversion term is not only relevant in addressing the transformation of data, but also for the designing the vertical grid of the model itself. In many applications the error arising from the transformation to the isobaric system could be tolerated if proven to be of unsystematic nature and small compared with the uncertainty of the reference value.

\section{Summary and conclusions}

We have introduced a method for transferring atmospheric model data to an isobaric coordinate system, based on the integration of model variables over finite pressure intervals. The chief advantage of this procedure over interpolation between model levels is that the mass-weighted integrals of variables are conserved. The application of an integral-conserving transformation not only to prognostic model variables, but to explicitly com-

Table 1. Comparative statistics for the vertically-integrated budget of specific enthalpy

\begin{tabular}{llcrrc}
\hline & exact & L21 & L16 & L9 \\
\hline flux divergence & mean & -181 & -179 & -175 & -179 \\
& standard deviation & 2124 & 2112 & 2079 & 2062 \\
& error standard deviation & & 42 & 145 & 226 \\
& correlation coefficient & & 1.000 & 0.998 & 0.995 \\
& mean & -273 & -273 & -272 & -301 \\
& standard deviation & 2424 & 2417 & 2415 & 2497 \\
& error standard deviation & & 31 & 96 & 687 \\
& correlation coefficient & & 1.000 & 0.999 & 0.961 \\
flux divergence-energy conversion conversion & mean & 92 & 94 & 97 & 122 \\
& standard deviation & 489 & 490 & 506 & 745 \\
& error standard deviation & & 33 & 101 & 618.6 \\
& correlation coefficient & & 0.998 & 0.980 & 0.565 \\
\hline
\end{tabular}

All parameters except the correlation coefficient are in units of $\mathrm{Wm}^{-2}$. 
puted and parameterized terms in relevant budget equations as well, would yield a very accurate representation of the budget in question, as defined by the assimilation system used. The accuracy of such a representation would be independent of the vertical resolution of the pressure coordinate system.

Unquestionably an integral-conserving transformation is a major advantage even in the traditional application of diagnosing budget residuals in the pressure frame using the isobaric representation of state fields, because it allows one to compute the vertical velocity from the transformed winds without any further adjustments. To draw the full benefit of this property, it is essential to express all advective terms in flux form and to take care to use exactly the same numerical formulation for the horizontal divergence operator whenever it appears. However, it must be kept in mind that any nonlinear term computed directly in the pressure frame as a nonlinear combination of state variables will always suffer from an error whose magnitude will depend on the structure of the output system.

We have explored this degradation in the context of the HIRLAM reanalysis project of the SMHI. It was found that the budget of dry enthalpy implied by the model data can be satisfactorily reproduced using transformed state variables, provided that the pressure grid is outlined with the necessary care. The most delicate components in this budget are the vertical advection and, even more, the energy conversion term. Besides the influence of the general assumptions, the vertical advection is affected by the definition of half level values in the isobaric frame. The sensitivity of the energy conversion term must stem from a high correlation in the vertical dimension between the small-scale variability of the temperature and the vertical velocity fields. This correlation is neglected when variables are assumed to be constant within pressure layers. For the energy conversion term, therefore, to the extent that one needs to recover this term exactly as defined by the model data, there seems to be no alternative to computing it in the model frame and transforming the result. On the other hand, the approximation obtained in the pressure frame provides a very reasonable picture of the threedimensional distribution of the field. The performance of the transformation and evaluation scheme was demonstrated explicitly only for the thermodynamic energy equation. However, since this budget is probably the most susceptible to changes of the vertical environment, we are confident that other budgets can be recovered essentially undistorted.

\section{Acknowledgments}

We wish to acknowledge Nils Gustafsson and the staff members of the re-analysis project at SMHI for providing the data and for valuable discussions. Thanks are extended also to Prof. Eero Holopainen for his encouragement and critical remarks during all stages of this work. The comments by two anonymous reviewers are also gratefully acknowledged. During the work Pierluigi Calanca was supported by the Swiss National Science Foundation through Grant No. 2-77-015-91, and Carl Fortelius partly by the Finnish Research Programme on Climate Change (SILMU) and partly by the EC Environment and Climate Research Programme (contract: ENV4-CT95-0072, Climatology and Natural Hazards) within the NEWBALTIC project.

\section{Appendix.}

\section{The boundary term $\lambda$}

According to Leibniz' rule, the integral of the mass flux divergence over a layer $p_{k+1 / 2}-p_{k-1 / 2}$ satisfies the relation:

$(\bar{\nabla} \cdot v)_{k}=\nabla \cdot \hat{v}_{k}+\lambda_{k}$,

where the boundary term $\lambda_{k}$ is given by:

$\lambda_{k}= \begin{cases}0 & \text { if } p_{k+1 / 2}<p_{\mathrm{s}}, \\ -v_{\mathrm{s}} \cdot \nabla p_{\mathrm{s}} & \text { if } p_{k-1 / 2}<p_{\mathrm{s}} \leqslant p_{k+1 / 2}, \\ 0 & \text { if } p_{k-1 / 2} \geqslant p_{\mathrm{s}},\end{cases}$

and $v_{\mathrm{s}}$ and $p_{\mathrm{s}}$ are the values of the horizontal wind at the surface and the surface pressure, respectively.

The conditions in (A.2) state that a non-zero boundary term arises whenever the layer under consideration is neither completely within the atmosphere nor completely below the ground. In a horizontally discrete environment this has to be 
verified not only at the grid point where the divergence is computed but also at all the grid points where the flux is specified. If the same condition is not satisfied at each one of these relevant points, none of the simple expressions in (A.2) is the correct one. We cope with this problem in the following way. In every column, we first determine those layers, distinguished henceforth by an index $k^{\prime}$, for which one and the same of the conditions in (A.2) is valid at all the relevant horizontal grid points. For these layers we compute the boundary term as in (A.2) and we form the sum:

$S_{1}=\sum_{k^{\prime}}\left(\widetilde{\nabla_{\mathrm{p}} \cdot \boldsymbol{v}}\right)_{k^{\prime}}=\nabla \cdot \sum_{k^{\prime}} \hat{v}_{k^{\prime}}+\sum_{k^{\prime}} \lambda_{k^{\prime}}$.

We then require our scheme to satisfy the discrete analogue of the relation

$\int_{0}^{p_{\mathrm{s}}} \nabla_{\mathrm{p}} \cdot v \mathrm{~d} p=\nabla \cdot \int_{0}^{p_{\mathrm{s}}} v \mathrm{~d} p-v_{\mathrm{s}} \cdot \nabla p_{\mathrm{s}}$ so that:

$S_{2}==\sum_{k=1}^{K}\left(\widehat{\nabla_{\mathrm{p}} \cdot \boldsymbol{v}}\right)_{k}=\nabla \cdot \sum_{k=1}^{K} \hat{v}_{k}-v_{\mathrm{s}} \cdot \nabla p_{\mathrm{s}}$

Provided that the lowest layer is chosen so that $p_{K+1 / 2}>p_{s}$ at every gridpoint, this is achieved if the integral of the flux divergence over all the problematic layers is equal to the difference $S_{2}-S_{1}$. We retrieve the layer-integrated flux divergence of the individual problematic layers simply by distributing $S_{2}-S_{1}$ among these layers in proportion to the actual layer thickness at the divergence point:

$\left.\widehat{\left(\nabla_{\mathrm{p}} \cdot \boldsymbol{v}\right.}\right)_{k}=\hat{\mathbf{1}}_{k}\left(\sum_{j \neq k^{\prime}} \hat{\mathbf{1}}_{j}\right)^{-1}\left(S_{2}-S_{1}\right), \quad \forall k \neq k^{\prime}$.

(A.1) and (A.6) together define the boundary term for the problematic layers as:

$\lambda_{k}=\left(\overline{\nabla_{\mathrm{p}} \cdot v}\right)_{k}-\nabla \cdot \hat{v}_{k}, \quad \forall k \neq k^{\prime}$.

\section{REFERENCES}

Andersson, T. and Gustafsson N., 1994. Coast of departure and coast of arrival. Two important concepts for the formation and structure of convective snow bands over seas and lakes. Mon. Wea. Rev. 122, 1036-1049.

Boer, G. 1982. Diagnostic equations in isobaric coordinates. Mon. Wea. Rev. 110, 1801-1820.

Gibson, J. K., Hernandez, A., Kållberg, P., Nomura, A. Serrano, E. and Uppala, S. 1994. The ECMWF re-analysis (ERA) project - plans and current status. Preprint for the 10th Conference on Numerical weather prediction, 18-22 July 1994, Portland, Oregon (American Meteorological Society).

Haltiner, G., and Williams, R. 1980. Numerical prediction and dynamic meteorology, 2nd edition John Wiley \& Sons, New York, 447 pp.

Holopainen, E.O. and Fortelius, C. 1986. Accuracy of estimates of atmospheric large-scale energy flux divergence. Mon. Wea. Rev. 114, 1910-1921.

Ljungemyr, P., Gustafsson N. and Omstedt, A. 1996. Parameterization of lake thermodynamics in a high resolution weather forecasting model. Tellus 48A, this issue.
Sass B. H. J. and Christensen, 1995. A simple framework for testing the quality of atmospheric limited-area models. Won. Wea. Rev., 123, 444-459.

Savijärvi, H. 1982. The mass balance in diagnostic studies; an example of analyzed and forecast data calculations. Tellus, 34A, 540-544.

Schubert, S. D., Rood, R. B. and Pfaendtner, J. 1993. An assimilated dataset for earth science applications. Bull. Amer. Meteor. Soc. 74, 2331-2342.

Simmons, A. and Burridge, D. 1981. An energy and angular momentum conserving vertical finite difference scheme and hybrid vertical coordinates. Mon. Wea. Rev. 109, 758-766.

Trenberth, K. 1991. Climate diagnostics from global analyses: Conservation of mass in ECMWF analyses. J. Climate 4, 707-722.

Trenberth, K. 1995. Truncation and use of modelcoordinate data. Tellus 47A, 287-303.

Trenberth, K. E., Hurrell, J. W. and Solomon, A. 1995. Conservation of mass in three dimensions in global analyses. J. Climate 8, 692-708. 